\title{
Spectral fitting of SDSS passive galaxies with $\alpha$-enhanced single stellar populations
}

\author{
Jean Michel Gomes ${ }^{1}$ and Paula Coelho ${ }^{2}$ \\ ${ }^{1}$ Centro de Astrofísica, Universidade do Porto, Rua das Estrelas, 4150-762 Porto, Portugal \\ ${ }^{2}$ Núcleo de Astrofísica Teórica, Universidade Cruzeiro do Sul, \\ R. Galvão Bueno 868, Liberdade, 01506-000, São Paulo, Brasil
}

email: jean@astro.up.pt

\begin{abstract}
The power of population synthesis as a mean to estimate the star-formation and chemical histories of galaxies has been well established in the last decade. The major developments were due to a huge avalanche of methods, codes and high-quality galaxy data sets, such as the $2 \mathrm{dF}, 6 \mathrm{dF}$ and SDSS surveys. Semi-empirical spectral synthesis allows for the decomposition of a galaxy spectrum in terms of linear combinations of base elements, i.e. Single Stellar Populations (SSPs) of different ages and metallicities, which are computed from evolutionary synthesis codes (BPASS, GALEV, GALAXEV, MILES, PÉGASE, etc...), containing distinct ingredients like: stellar library, evolutionary tracks, metallicities and Initial Mass Function. In general, they have solar-scaled relative abundances, but this is about to change with the unfolding of new $\alpha$-enhanced SSP models (Coelho et al. 2007). However, passive galaxies have some spectral features corresponding to "enhanced-ratios" ([E/Fe]), like $\mathrm{O}, \mathrm{Ne}, \mathrm{Si}, \mathrm{S}, \mathrm{Mg}, \mathrm{Na}, \mathrm{C}$ and $\mathrm{N}$ over Fe that are not well modeled using solar-scaled SSPs (Trager et al. 2000), leading to residuals between observed and modeled spectra, which also correlate with the velocity dispersion $\left(\sigma_{\star}\right)$ and stellar mass $\left(M_{\star}\right)$ : Massive galaxies exhibit a larger [E/Fe] discrepancy than less massive ones. This result can be interpreted as a signature of distinct previous star-formation efficiencies in passive galaxies, leading to distinctive ratios of type Ia and II SNe.

We have applied the STARLIGHT spectral synthesis code (Cid Fernandes et al. 2005) to a sample of $\sim 1000$ passive galaxies from the SDSS DR7 with a $\mathrm{S} / \mathrm{N}$ at the continuum $\geqslant 20$ to investigate possible enhancements in the derived $[\mathrm{E} / \mathrm{Fe}]$ ratios. Three sets of SSPs based on Coelho et al. (2007) theoretical models and Walcher et al. (2009) prescriptions were computed for $[\alpha / \mathrm{Fe}]=0.0,[\alpha / \mathrm{Fe}]=0.2$ and $[\alpha / \mathrm{Fe}]=0.4$. Our aim is to determine: (1) the quality of the fits, (2) the mean stellar age and metallicity distributions, and (3) the star-formation history of passive galaxies.

Using $[\alpha / \mathrm{Fe}]=0.0 \mathrm{SSPs}$, we have identified the strongest residuals in the $\mathrm{CN}(4142.125-$ $4177.125 \AA)$, Na D $(5876.875-5909.375 \AA)$ and $\mathrm{Mg}(5069.125$ - 5196.625 $\AA$ ) bands. On the other hand, $[\alpha / \mathrm{Fe}]=0.2$ and $[\alpha / \mathrm{Fe}]=0.4$ SSP models tend to reproduce better the $\mathrm{Mg}$ band, as compared to solar-scaled SSPs $([\alpha / \mathrm{Fe}]=0.0)$. The residuals are decreased by $1.77 \AA([\alpha / \mathrm{Fe}]=0.2)$ and $2.92 \AA([\alpha / \mathrm{Fe}]=0.4)$. However, as expected, these $\alpha$-enhanced models lead to worse fits for the $\mathrm{CN}$ and Na D bands. These residuals may even reach up to $2.08 \AA(\mathrm{CN})$ and $4.20 \AA(\mathrm{Na} \mathrm{D})$, using $[\alpha / \mathrm{Fe}]=0.2 \mathrm{SSPs}$ and $2.28 \AA(\mathrm{CN})$ and $7.94 \AA(\mathrm{Na} \mathrm{D})$, using $[\alpha / \mathrm{Fe}]=0.4$ SSPs.

In terms of mean stellar ages and metallicities, we obtain non-negligible biases in both quantities when we compare the solar-scaled SSPs with $\alpha$-enhanced ones, which tend to have mean stellar ages by $0.12 \operatorname{dex}([\alpha / \mathrm{Fe}]=0.2)$ and $0.14 \operatorname{dex}([\alpha / \mathrm{Fe}]=0.4)$ higher and mean stellar metallicities by $0.1 \operatorname{dex}([\alpha / \mathrm{Fe}]=0.2)$ and $0.2 \operatorname{dex}([\alpha / \mathrm{Fe}]=0.4)$ lower.
\end{abstract}

Keywords. galaxies: evolution, galaxies: formation, galaxies: stellar content 


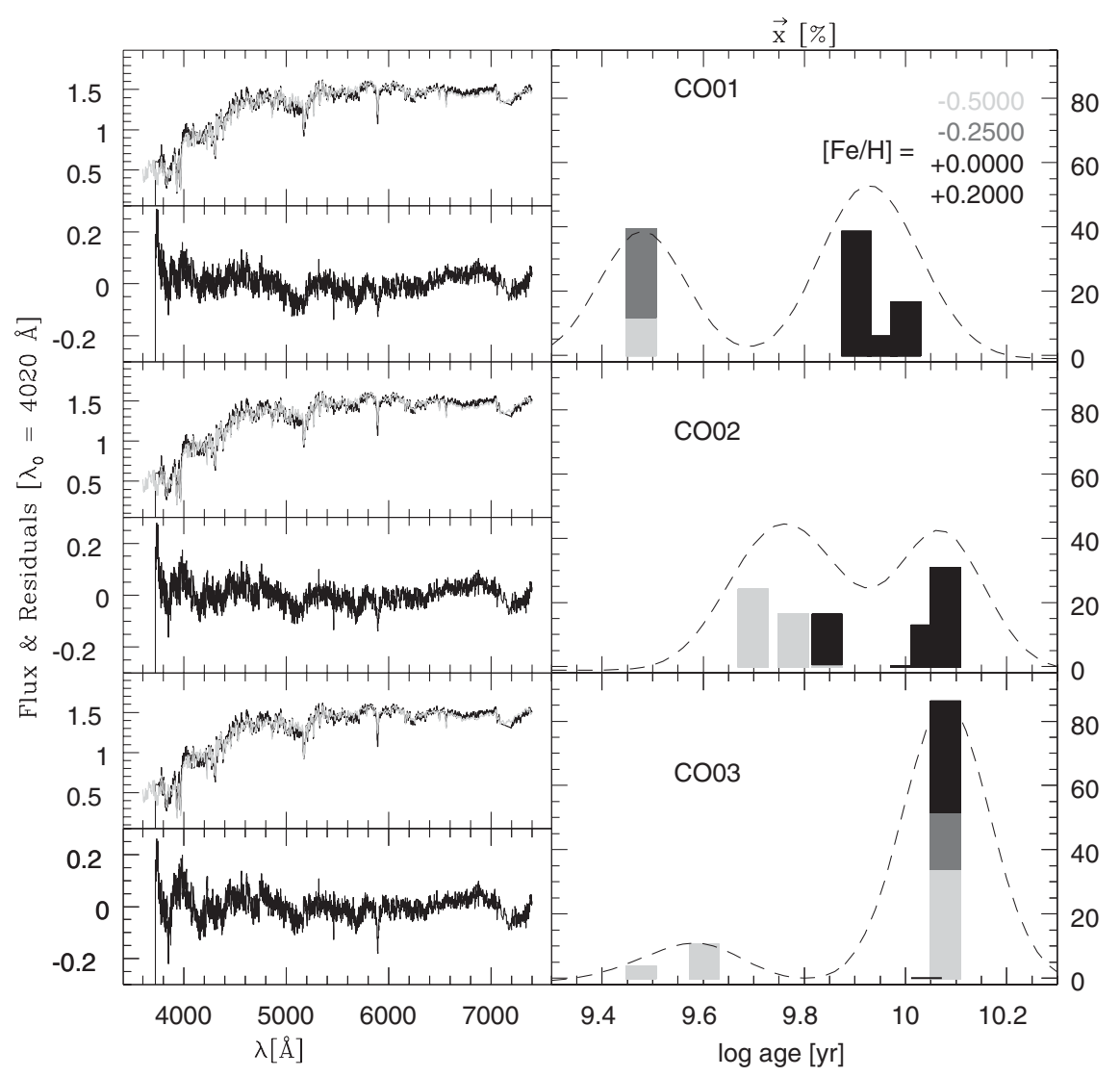

Figure 1. Spectral fit to the SDSS passive galaxy J100329.97+372734.6 using three different sets of SSPs with the following $\alpha$-enhancement ratios: CO01 $([\alpha / \mathrm{Fe}]=0.0), \mathrm{CO} 02([\alpha / \mathrm{Fe}]=0.2)$ and $\mathrm{CO} 03([\alpha / \mathrm{Fe}]=0.4)$, spanning 10 distinct ages from 3 to $12 \mathrm{Gyr}$ and 4 different metallicities $([\mathrm{Fe} / \mathrm{H}]=-0.50,-0.25,+0.00 \&+0.20)$. Left panels: Observed (black) and modeled (grey) spectrum with the corresponding residuals for CO01, CO02 and CO03 (from top to bottom). The fits are equally good in terms of their reduced $\chi^{2}$. The corresponding Star Formation Histories (SFHs) are displayed in the right panels. The smoothed SFHs (re-scaled) are shown (black dashed line) with a Gaussian FWHM of 0.2 dex, further illustrating the tendency for higher mean stellar ages with increasing $[\alpha / \mathrm{Fe}] \mathrm{SSP}$ abundances.

\section{Sample of passive galaxies}

We have applied STARLIGHT to $\sim 1000$ passive galaxies, lacking nebular emission, from the SDSS DR7, using three different libraries with distinct $\alpha$-enhanced SSP models: CO01 $([\alpha / \mathrm{Fe}]=0.0), \mathrm{CO} 02([\alpha / \mathrm{Fe}]=0.2)$ and $\mathrm{CO} 03([\alpha / \mathrm{Fe}]=0.4)$, spanning a range in ages from 3 to $12 \mathrm{Gyr}$, and 4 metallicities $([\mathrm{Fe} / \mathrm{H}]=-0.50,-0.25,+0.00 \&+0.20)$. In Fig. 1 we show an example for the spectral fits and the corresponding Star Formation Histories (SFH) to the SDSS passive galaxy J100329.97+372734.6 using CO01, CO02 and CO03 SSPs.

\section{Mean stellar age and metallicity}

Fig. 2 shows the degeneracy diagram of age versus metallicity, showing a clear trend for a higher mean stellar age and a lower mean stellar metallicity with increasing $\alpha$-element enhancement in model SSPs. 


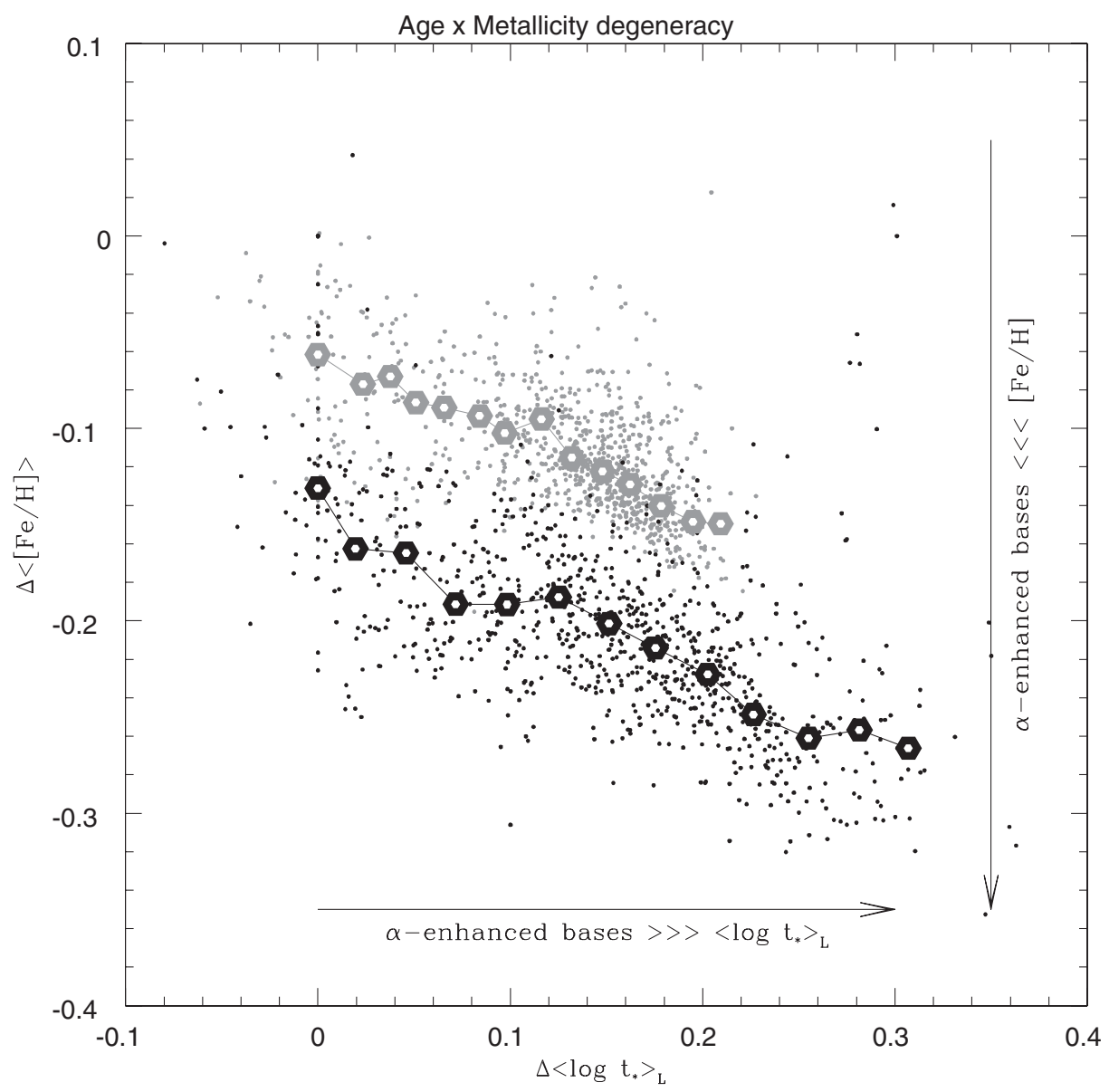

Figure 2. The degeneracy diagram of Age vs Metallicity. We show the difference in mean stellar age and mean stellar metallicity for $\mathrm{CO} 02[\alpha / \mathrm{Fe}=0.2]$ (grey) / $\mathrm{CO} 03[\alpha / \mathrm{Fe}=0.3]$ (black) compared to $\mathrm{CO} 01[\alpha / \mathrm{Fe}=0.0]$. The hexagons correspond to the median values: top for $\mathrm{CO} 02-\mathrm{CO} 01$ and bottom for CO03-CO01. A clear trend for a higher mean stellar age and a lower mean stellar metallicity with increasing $\alpha$-element enhancement is apparent.

\section{Acknowledgements}

J. M. Gomes is supported by a Post-Doctoral grant, funded by FCT/MCTES (Portugal) and POPH/FSE (EC). Special thanks also to Privatdozent Dr. P. Papaderos for making suggestions and revising the manuscript.

\section{References}

Cid Fernandes R. et al. 2005, MNRAS, 358, 363

Coelho P. et al. 2007, MNRAS, 382, 498

Lee H.-C. et al. 2009, AJ, 138, 1442

Trager S. C. et al. $2000, A J, 120,165$

Walcher C. J. et al. 2009, MNRAS, 398, 44

Worthey G., Faber S. M., \& Gonzalez J. J. 1992, ApJ, 398, 69

Worthey G. 1994, ApJ, 95, 107 